ISSN: 1679-3013

D.O.I.: 10.5914/to.2011.0077

\title{
AVALIAÇÃO TEMPORAL DA ACUMULAÇÃO DE ELEMENTOS TRAÇO NO CAMARÃO- ROSA Farfantepenaeus paulensis NO ESTUÁRIO DA LAGOA DOS PATOS, RS, BRASIL
}

\author{
Janice Goris GARCIA \\ Luis Felipe Hax NIENCHESKI
}

Recebido em: $31 / 03 / 2011$

Aceito em: 05/11/2012

\section{RESUMO}

Este estudo objetivou avaliar as concentrações de $\mathrm{As}, \mathrm{Cd}, \mathrm{Cr}, \mathrm{Cu}, \mathrm{Fe}, \mathrm{Mn}, \mathrm{Ni}, \mathrm{Pb}$ e Zn no camarão-rosa Farfantepenaeus paulensis associadas às flutuações nas condições hidrológicas estuarinas, no período de 2006 à 2010. Os organismos foram coletados anualmente e as concentrações de metais foram determinadas no organismo inteiro e na fração abdominal, por espectrometria de absorção atômica.Teores mais elevados de metais foram encontrados no camarão analisado por inteiro quando comparado à fração abdominal. As concentrações de As e $\mathrm{Pb}$ analisadas nas duas formas, e de $\mathrm{Mn}$ para o camarão inteiro, revelaram valores mais elevados em anos de maior ocorrência de pluviosidade, associada ao fenômeno El Nino. Contrariamente, as maiores concentrações de Cu no camarão inteiro foram observadas quando o estuário apresentou-se dominado por água marinha, característico de períodos de La Niña. Os demais metais analisados nos organismos não estiveram relacionados com os parâmetros ambientais contemplados neste estudo (material em suspensão e salinidade).

Palavras-chave: crustáceos; elementos traço; condições hidrológicas.

\section{ABSTRACT}

This study aimed to evaluate the concentrations of $\mathrm{As}, \mathrm{Cd}, \mathrm{Cr}, \mathrm{Cu}, \mathrm{Fe}, \mathrm{Mn}, \mathrm{Ni}, \mathrm{Pb}$ and $\mathrm{Zn}$ in Farfantepenaeus paulensis associated with fluctuations in estuarine hydrological conditions from 2006 to 2010. Organisms were collected annually and metal concentrations were determined in whole shrimp and edible part by Atomic Absorption Spectrometry. The whole shrimp (soft tissue and shell) under analysis generally exhibited higher concentrations of metals that the edible part. Both forms under analysis showed high levels of $\mathrm{As}$ and $\mathrm{Pb}$ in years of high occurrence of rainfall associated with $\mathrm{El}$ Niño effect. It was also observed for $\mathrm{Mn}$ in the whole shrimps analyzed. In contrast, the highest concentration of $\mathrm{Cu}$ in the whole shrimp were observed when the estuary was dominated by seawater, characteristic of La Niña periods. The other elements analyzed in the organisms were not related to the environmental parameters considered in this study (suspended matter and salinity).

Keywords: crustaceans, trace elements, hydrologic conditions

\section{INTRODUÇÃO}

O camarão- rosa Farfantepenaeus paulensis é uma espécie nativa do Oceano Atlântico Ocidental, que se distribui desde Ilhéus na Bahia (Brasil -14050'S) até o Mar del Plata (Argentina - 38³5'S) (D'Incao, 1995). Seu ciclo de vida apresenta uma fase oceânica, onde ocorre a reprodução e desenvolvimento larval, e outra estuarina, caracterizada pelo desenvolvimento de juvenis até a migração para o oceano, três a quatro meses após sua entrada no estuário (Iwai, 1978; D’Incao, 1991).

O estuário da Lagoa dos Patos é considerado o mais importante criadouro desta espécie no Brasil, que se constitui em um dos principais recursos pesqueiros da região Sul, devido ao seu alto valor comercial. A intensa pesca efetuada sobre os juvenis contribui para a redução do estoque adulto, que é considerado sobre-explotado desde a década de 80 e colapsado no início da década atual (D'Incao et al. 2002). 
Além do esforço de pesca, defasagens na produção do camarão também estão associadas à degradação ambiental via aterros, dragagem, assoreamento e poluentes (Altmayer,1999). Deste último, os metais são preocupantes, pela sua persistência no ambiente e a tendência a se concentrarem nos organismos.

Crustáceos têm sido amplamente utilizados como indicadores biológicos de poluição costeira e, na avaliação da influência de metais no ambiente marinho. Apresentam potencial para serem utilizados como sentinelas, uma vez que podem acumular metais por absorção através das brânquias ou por consumo de sedimentos e detritos contaminados. Adicionalmente, informações de concentrações de metais em seus tecidos são potencialmente úteis considerando sua toxicidade e a saúde pública, tendo em vista seu amplo consumo pela população.

O estuário da Lagoa dos Patos vem sofrendo uma forte pressão ambiental, devido à crescente urbanização do município de Rio Grande, crescimento e diversificação das atividades industriais e portuárias, e ao recente desenvolvimento de um pólo naval. Neste estudo foram avaliadas as concentrações dos metais $\mathrm{As}, \mathrm{Cd}, \mathrm{Cr}, \mathrm{Cu}, \mathrm{Fe}, \mathrm{Mn}, \mathrm{Ni}, \mathrm{Pb}$ e Zn no camarão-rosa Farfantepenaeus paulensis, coletados no estuário da Lagoa dos Patos durante cinco anos consecutivos.

A Lagoa dos Patos situa-se entre $30^{\circ}$ e $32^{\circ}$ de latitude sul e representa a maior lagoa costeira do Atlântico Sul Ocidental (Garcia \& Vieira, 2001). Recebe aporte de água doce dos rios da parte norte da planície costeira do Rio Grande do Sul e dos rios afluentes da Lagoa Mirim. Na maior parte do tempo sua área estuarina abrange uma área que vai desde a barra do Rio Grande até uma linha imaginária que liga a Ponta da Feitoria $\left(31^{\circ} 41^{\prime} \mathrm{S}\right.$ e $52^{\circ} 02^{\prime} \mathrm{W}$ ) à Ponta dos Lençóis ( $31^{\circ} 48^{\prime} \mathrm{S}$ e $51^{\circ} 52^{\prime} \mathrm{W}$ ) (Figura 1 ) (Möller \& Fernandes, 2010).

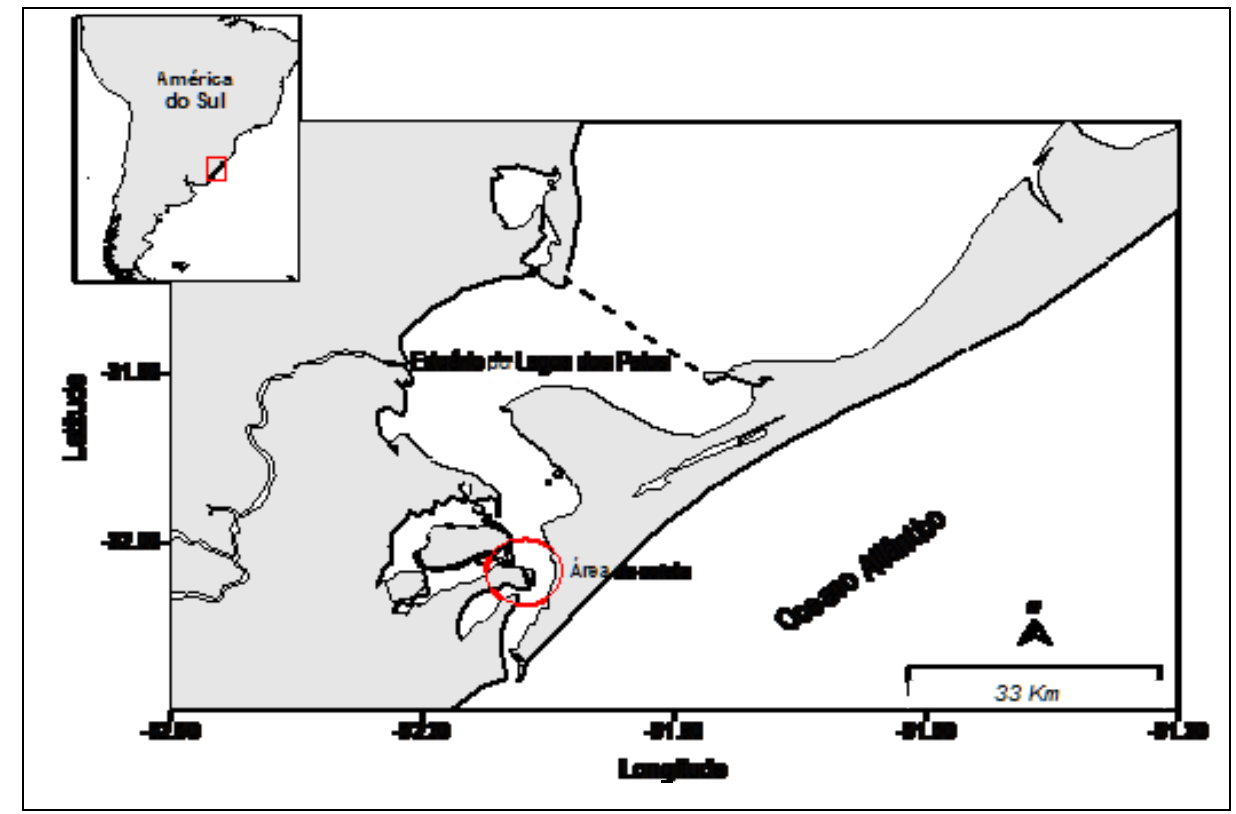

Figura 1 - Área de estudo e local de coleta dos camarões Farfantepenaeus paulensis.

Nesta região o efeito da maré tem importância secundária. A relação entre descarga fluvial e ação dos ventos altera profundamente a circulação das águas lagunares e, consequentemente, os processos de dessalinização ou salinização, que podem durar por vários meses. A salinidade no estuário decresce de forma exponencial com o aumento da descarga fluvial (Möller et al. 1991; Fernandes 2001; Fernandes et al. 2002). Este aumento associa-se diretamente a períodos de grande ocorrência de pluviosidade, associados ao fenômeno El Niño (Berlato \& Fontana, 2003). A passagem de frentes meteorológicas eleva o nível do mar na costa sob efeito de ventos Sudoeste (SO), forçando águas marinhas para o interior da laguna, em períodos de descarga fluvial fraca ou moderada, característico de períodos de La Niña, quando a precipitação pode ficar 
abaixo da média (Berlato \& Fontana, 2003), favorecendo a intensa salinização da Lagoa dos Patos.

Variações na intensidade de salinização deste ambiente implicam no maior ou menor tempo de residência de substâncias naturais ou introduzidas por atividade humana, e podem refletir em variações na acumulação dos metais pelos organismos.

Embora o estuário da Lagoa dos Patos tenha sido alvo de diversos estudos ambientais (Ustra, 2001; Vilas Boas, 1990; Baumgarten \& Niencheski, 1990), bem como de estudos que mostram os efeitos de metais em organismo estuarinos, falta muito conhecimento desses efeitos combinados com fatores ambientais como salinidade e temperatura (Barbieri et al., 2005), e não consta na literatura científica estudos que demonstrem os reflexos das variações hidrológicas interanuais deste estuário na acumulação de metais no camarão-rosa.

Dessa forma, este estudo teve como objetivo principal avaliar as concentrações de metais no camarão-rosa Farfantepenaeus paulensis associadas às flutuações nas condições hidrológicas no estuário da Lagoa dos Patos, durante o período de 2006 à 2010.

\section{MATERIAL E MÉTODOS}

\section{Coleta e processamento das amostras}

Entre os anos de 2006 e 2010 foram amostrados 86 camarões-rosa na porção sul do estuário da Lagoa dos Patos, com o auxílio de uma rede de arrasto. As coletas foram realizadas anualmente, no mês de fevereiro, o qual está inserido no período de safra comercial do camarão-rosa (de fevereiro à maio). Após cada coleta, os organismos foram pesados e foi determinado o comprimento total, individualmente. Os camarões foram separados em dois grupos: com organismos inteiros (com casca) e com organismos sem casca (somente a fração abdominal). Cada grupo de organismos foram distribuídos em três placas de Petry, formando amostras compostas com aproximadamente 10 organismos em cada. Apenas no ano de 2010 foram utilizadas somente uma amostra composta para cada grupo (inteiro e limpo), devido ao número amostral reduzidoAs amostras foram secas em estufa à $60^{\circ} \mathrm{C}$, resultando em uma umidade média de $78,7 \%$ para o camarão inteiro e 79,7\% para o limpo.

Foi pesado em torno de $0,5 \mathrm{~g}$ - peso seco, de cada amostra que sofreu digestão ácida com $5 \mathrm{~mL} \mathrm{HNO}_{3}(65 \%)^{\circledR}$ em uma chapa aquecedora a $100^{\circ} \mathrm{C}$ até total dissolução da amostra. O material foi avolumado $25 \mathrm{~mL}$ com água de alta pureza sendo retirada uma alíquota para análise (Niencheski, 2006).

Os dados de material em suspensão foram obtidos de relatórios técnicos-científicos disponibilizados por SUPRG (2010), e representam as análises da água estuarina coletada em 11 pontos amostrais fixos distribuídos ao longo do estuário.

A salinidade foi representada utilizando os valores mínimos e máximos encontrados para o período de verão de cada ano, com base em Pereira (2010), com exceção do verão de 2010 que foi obtido de SUPRG (2010).

A determinação da concentração dos metais $\mathrm{As}, \mathrm{Cd}, \mathrm{Cr}, \mathrm{Mn}, \mathrm{Ni}$ e $\mathrm{Pb}$ nos organismos, bem como de todos os metais em água foi feita por Espectrometria de Absorção Atômica com Forno de Grafite (ZEISS -AAS 5) e a dos metais Cu, Fe e Zn nos organismos foi feita por Espectrometria de Absorção Atômica de Chama (CG AA 7000 SBC).Para assegurar a qualidade analítica, foram utilizados em cada batelada de análises, o material certificado de referência IAEA-350 (Tuna-Fish, músculo), seguindo o mesmo procedimento metodológico aplicado nas amostras. A recuperação média do material de referência não ultrapassou uma variação de $10 \%$ do valor certificado.

\section{Análises estatísticas dos resultados}

Primeiramente, foram testadas a normalidade dos dados através do teste ShapiroWilk e a homogeneidade das variâncias, através do teste Cochran. Como todos os metais 
apresentaram normalidade e homocedasticidade após as transformações matemáticas indicadas por Zar (1984), utilizou-se ANOVA bifatorial na comparação de cada elemento entre anos e tecidos, para os organismos, e ANOVA de uma via para as comparações interanuais dos teores de metais na água. Em ambas, foi utilizado o teste de Tukey a posteriori.

\section{RESULTADOS}

A Tabela 1 mostra os valores médios das concentrações de metal total, do material em suspensão (MS) e, valores máximos e mínimos de salinidade para o período de verão, em cada ano.

Tabela 1: Concentrações de material em suspensão (valores médios \pm desvio padrão) e Salinidade (valores mínimos e máximos) na água de fundo coletada em 11 pontos amostrais no estuário da Lagoa dos Patos.

\begin{tabular}{c|c|c|c|c|c}
\hline Ano & $\mathbf{2 0 0 6}$ & $\mathbf{2 0 0 7}$ & $\mathbf{2 0 0 8}$ & $\mathbf{2 0 0 9}$ & $\mathbf{2 0 1 0}$ \\
\hline M.S. & $36,21 \pm 12,2$ & $75,78 \pm 35,7$ & $149,3 \pm 80$ & $75,9 \pm 28,5$ & $77 \pm 40$ \\
\hline Salinidade & $10-25$ & $20-30$ & $5-20$ & $20-30$ & $2,5-12,2$ \\
\hline
\end{tabular}

A Tabela 2 mostra os resultados encontrados na análise do material de referência bem como o Limite de Detecção para cada elemento.

Tabela 2: Limite de deteç̧ão dos metais $\left(\mu \mathrm{g} \cdot \mathrm{L}^{-1}\right)$, valores certificados $(\mu \mathrm{g} / \mathrm{g}-$ peso seco) e valores encontrados ( $\mu \mathrm{g} / \mathrm{g}$ - peso seco) do material de referência IAEA 350 (valores médios, mínimos e máximos entre parênteses) são apresentados.

\begin{tabular}{c|ccc}
\hline & Valores certificados & Valores encontrados $(\mathbf{n}=5)$ & Limite de deteç̧ão \\
\hline $\mathbf{A s}$ & $3,73(2,5-5,71)$ & $1,53(1,27-3,53)$ & 0,17 \\
\hline $\mathbf{C d}$ & $0,032(0,018-0,05)$ & $0,034(0,033-0,04)$ & 0,01 \\
\hline $\mathbf{C r}$ & $0,75(0,55-1,01)$ & $0,73(0,48-0,79)$ & 0,39 \\
\hline $\mathbf{C u}$ & $2,83(2,55-3,1)$ & $2,79(2,52-2,79)$ & 0,02 \\
\hline $\mathbf{F e}$ & $72,1(66,7-77,3)$ & $75,61(68,91-86,1)$ & 0,19 \\
\hline $\mathbf{M n}$ & $0,6(0,52-0,74)$ & $0,68(0,42-0,96)$ & 0,03 \\
\hline $\mathbf{N i}$ & $0,46(0,32-1,13)$ & $0,34(0,33-0,4)$ & 0,34 \\
\hline $\mathbf{P b}$ & $0,48(0,2-0,86)$ & $0,49(0,46-0,53)$ & 0,04 \\
\hline $\mathbf{Z n}$ & $17,4(16,6-18,5)$ & $17,61(15,98-20,01)$ & 0,002 \\
\hline
\end{tabular}

\section{Metais analisados no camarão-rosa}

A Tabela 3 apresenta os valores médios de metais encontrados para o camarão inteiro e limpo, para os 5 anos de amostragens. 
GARCIA, J. G.; NIENCHESKI, L. F. H. Avaliação temporal da acumulação de elementos traço no camarão-rosa Farfantepenaeus paulensis no estuário da Lagoa dos Patos, RS, Brasil.

Tabela 3: Concentrações de metais no camarão-rosa Farfantepenaeus paulensis. Valores médios ( $\mu \mathrm{g} / \mathrm{g}$ - peso seco) \pm desvio-padrão, valores mínimos e máximos entre parênteses são apresentados.

\begin{tabular}{|c|c|c|c|c|c|c|c|c|c|}
\hline & As & Cd & $\mathrm{Cr}$ & $\mathrm{Cu}$ & $\mathrm{Fe}$ & Mn & $\mathrm{Ni}$ & $\mathrm{Pb}$ & $\mathrm{Zn}$ \\
\hline \multicolumn{10}{|l|}{ Inteiro } \\
\hline 2006 & $\begin{array}{c}0,73 \pm 0,16 \\
(0,62-0,84)\end{array}$ & $\begin{array}{c}0,28 \pm 0,03 \\
(0,26-0,30) \\
\end{array}$ & $\begin{array}{c}0,30 \pm 0,33 \\
(0,07-0,54)\end{array}$ & $\begin{array}{c}78,25 \pm 2,41 \\
(76,55-79,96) \\
\end{array}$ & $\begin{array}{r}127,37 \pm 99,37 \\
(57,10-197,64) \\
\end{array}$ & $\begin{array}{l}6,23 \pm 0,86 \\
(5,62-6,84) \\
\end{array}$ & $\begin{array}{c}0,14 \pm 0,04 \\
(0,12-0,17) \\
\end{array}$ & A.D. & $\begin{array}{c}52,91 \pm 6,32 \\
(48,44-57,38) \\
\end{array}$ \\
\hline 2007 & < L.D. & 0,2 & 0,36 & 52,78 & 30,94 & 6,27 & 0,49 & 0,02 & 42,12 \\
\hline 2008 & $\begin{array}{c}1 \pm 0,2 \\
(0,76-0,88)\end{array}$ & $\begin{array}{c}0,23 \pm 0,05 \\
(0,19-0,29)\end{array}$ & $\begin{array}{c}0,25 \pm 0,04 \\
(0,21-0,28)\end{array}$ & $\begin{array}{c}13,9 \pm 1,58 \\
(12,14-15,17)\end{array}$ & $\begin{array}{c}291,77 \pm 229,55 \\
(26,48-122,1)\end{array}$ & $\begin{array}{c}3,61 \pm 3,25 \\
(6,37-19,49)\end{array}$ & $\begin{array}{l}2,04 \pm 0,34 \\
(1,73-2,4)\end{array}$ & $\begin{array}{c}1,66 \pm 0,19 \\
(1,52-1,88)\end{array}$ & $\begin{array}{c}43,16 \pm 2,5 \\
(40,95-45,88)\end{array}$ \\
\hline 2009 & $\begin{array}{c}0,03 \pm 0,02 \\
(0-0,04)\end{array}$ & $\begin{array}{c}0,2 \pm 0,14 \\
(0-0,29)\end{array}$ & $\begin{array}{c}0,13 \pm 0,16 \\
(0-0,33)\end{array}$ & $\begin{array}{c}24,88 \pm 2,92 \\
(22,17-28,51)\end{array}$ & $\begin{array}{c}141,14 \pm 63,51 \\
(85,31 \pm 201,51)\end{array}$ & $\begin{array}{l}0,76 \pm 0,07 \\
(0,72-0,86)\end{array}$ & $\begin{array}{c}0,72 \pm 0,55 \\
(0-1,2)\end{array}$ & $\begin{array}{c}0,12 \pm 0,16 \\
(0-0,36)\end{array}$ & $\begin{array}{l}48,74 \pm 10,5 \\
(37,9-61,98)\end{array}$ \\
\hline 2010 & 6,85 & 0,21 & $<$ L.D & 10,59 & 94,8 & 18,73 & $<$ L.D & 0,16 & 45,97 \\
\hline \multicolumn{10}{|c|}{ Músculo } \\
\hline 2006 & $\begin{array}{c}0,60 \pm 0,1 \\
(0,53-0,67)\end{array}$ & $\begin{array}{c}0,08 \pm 0,05 \\
(0,05-0,11)\end{array}$ & $\begin{array}{c}0,26 \pm 0,32 \\
(0,04-0,49)\end{array}$ & $\begin{array}{c}22,2 \pm 7,96 \\
(16,59-27,84)\end{array}$ & $\begin{array}{c}24,56 \pm 19,73 \\
(10,61-38,51)\end{array}$ & $\begin{array}{l}1,58 \pm 0,25 \\
(1,41-1,75)\end{array}$ & $\begin{array}{l}0,05 \pm 0,01 \\
(0,04-0,06)\end{array}$ & A.D. & $\begin{array}{r}39,61 \pm 18,64 \\
(26,43-52,79)\end{array}$ \\
\hline 2007 & $<$ L.D. & 0,07 & 0,15 & 40,51 & 18,17 & 4,26 & 0,1 & 0,01 & 33,92 \\
\hline 2008 & $\begin{array}{c}0,83 \pm 0,07 \\
(0,86-1,22)\end{array}$ & $\begin{array}{c}0,12 \pm 0,04 \\
(0,09-0,17)\end{array}$ & $\begin{array}{c}0,12 \pm 0,04 \\
(0,09-0,16)\end{array}$ & $\begin{array}{c}7,38 \pm 1,48 \\
(5,73-8,59)\end{array}$ & $\begin{array}{c}80,91 \pm 49,16 \\
(57,21-515,96)\end{array}$ & $\begin{array}{l}14,52 \pm 7,11 \\
(1,59-7,36)\end{array}$ & $\begin{array}{l}0,53 \pm 0,11 \\
(0,40-0,59)\end{array}$ & $\begin{array}{c}1,13 \pm 0,05 \\
(1,07-1,17)\end{array}$ & $\begin{array}{c}37,21 \pm 1,64 \\
(35,77-38,98)\end{array}$ \\
\hline 2009 & $\begin{array}{c}0,14 \pm 0,07 \\
(0,04-0,19)\end{array}$ & $\begin{array}{l}0,04 \pm 0,01 \\
(0,03-0,06)\end{array}$ & $\begin{array}{c}0,05 \pm 0,07 \\
(0-0,15)\end{array}$ & $\begin{array}{c}166,6 \pm 19,35 \\
(144,25-191,35)\end{array}$ & $\begin{array}{c}72,66 \pm 41,7 \\
(12,71-106,93)\end{array}$ & $\begin{array}{l}0,45 \pm 0,13 \\
(0,31-0,6)\end{array}$ & $\begin{array}{l}0,25 \pm 0,13 \\
(0,16-0,44)\end{array}$ & $\begin{array}{c}0,18 \pm 0,08 \\
(0,11-0,27)\end{array}$ & $\begin{array}{l}35,03 \pm 11,82 \\
(17,8-44,64)\end{array}$ \\
\hline 2010 & 7,65 & 0,04 & $<$ L.D. & 4,97 & 40,98 & 3,29 & $<L . D$ & 0,03 & 23,52 \\
\hline
\end{tabular}

*A.D.: Ausência de dados L.D.: Limite de Detecção

Em geral, as concentrações dos metais foram mais altas no camarão inteiro, sendo essas diferenças significativas $(p<0,5)$ para os metais $\mathrm{Cd}$ e $\mathrm{Fe}$ em todos os anos de amostragens; $\mathrm{Ni}$ e $\mathrm{Pb}$ somente nos camarões amostrados em 2008; para o Mn, neste ano, e em 2010 e, para o Cu, no ano de 2009 (Figura 2).
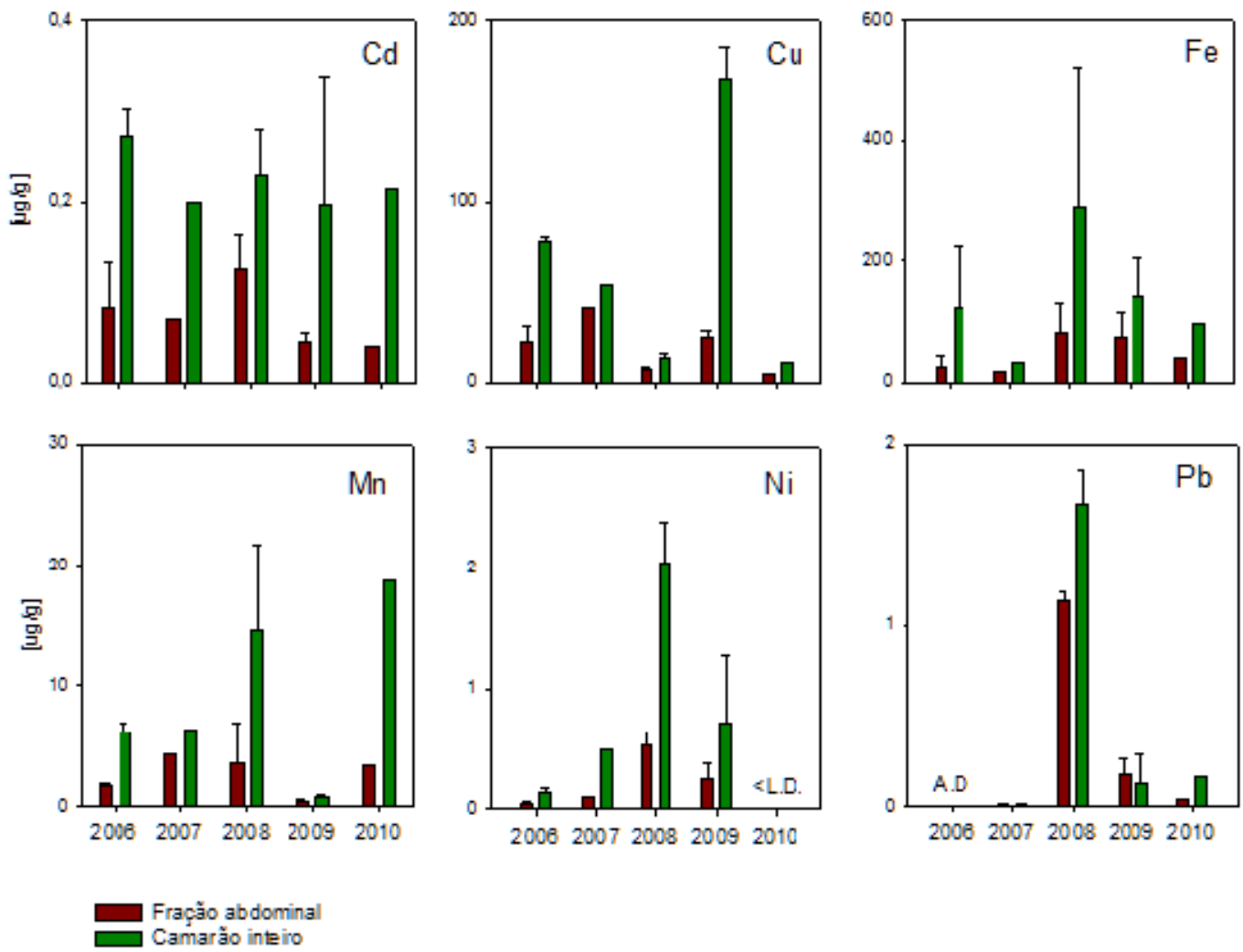

Figura 2 - Concentrações de metais ( $\mu \mathrm{g} / \mathrm{g}$ - peso seco) no camarão rosa, entre os anos de 2006 e 2010.(A.D.: Ausência de dados, L.D.: Limite de detecção). 


\section{Comparações interanuais}

Além das diferenças entre as formas analisadas, também foram observadas diferenças interanuais significativas.

Com relação à fração limpa analisada, concentrações significativamente maiores de $\mathrm{Pb}$, no ano de 2008 e de As, em 2010 ( $p<0,5)$ (Figura 3).

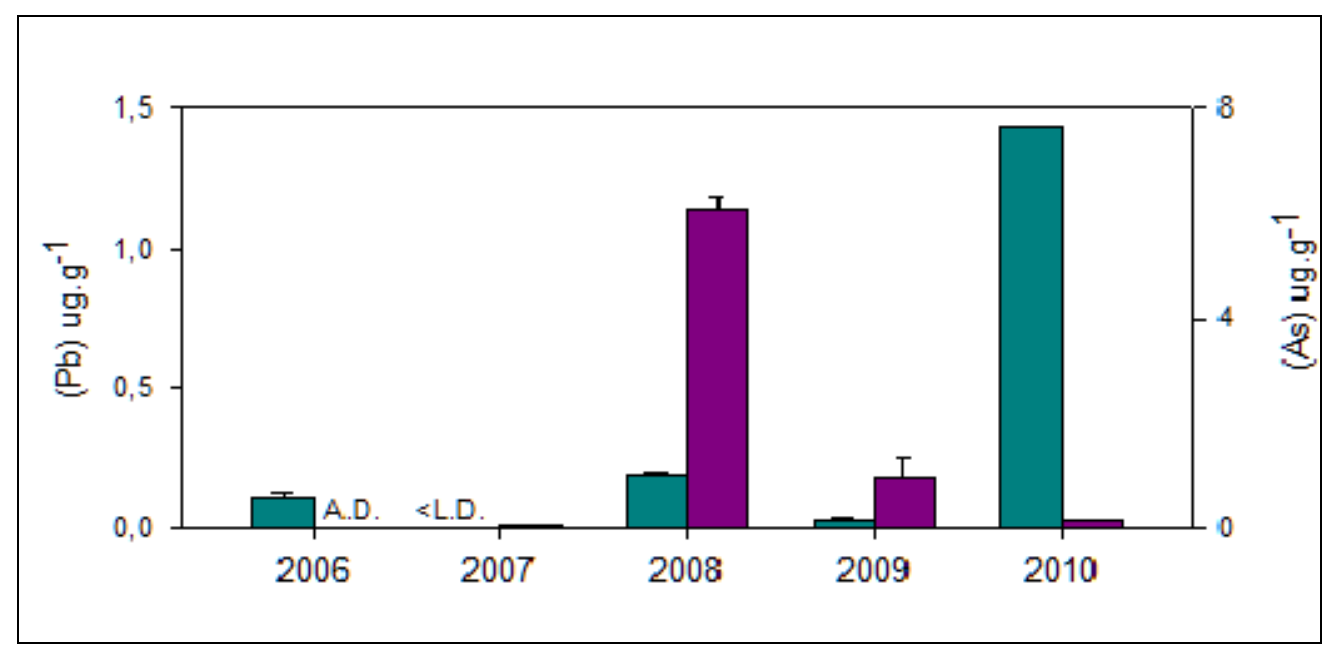

Figura 3 - Concentrações $\left(\mu \mathrm{g} \cdot \mathrm{g}^{-1}\right.$ - peso seco) de $\mathrm{Pb}$ (roxo) e As (verde) na fração limpa analisada do camarão-rosa, entre os anos de 2006 e 2010. (A.D.: Ausência de dados, L.D.: Limite de detecção).

Já os exemplares inteiros exibiram o mesmo comportamento que a fração limpa, com relação aos metais $\mathrm{Pb}$ e As, com maiores concentrações em 2008 e 2010, respectivamente $(p<0,05)$ (Figura 4$)$. Além desses metais, maiores concentrações de $\mathrm{Ni}$ e Mn foram observadas nos camarões coletados em 2008. Os amostrados em 2009 apresentaram maiores teores de $\mathrm{Cu}$ e, em 2010 novamente apresentaram teores elevados de $M n(p<0,05)$ (Figura 4).
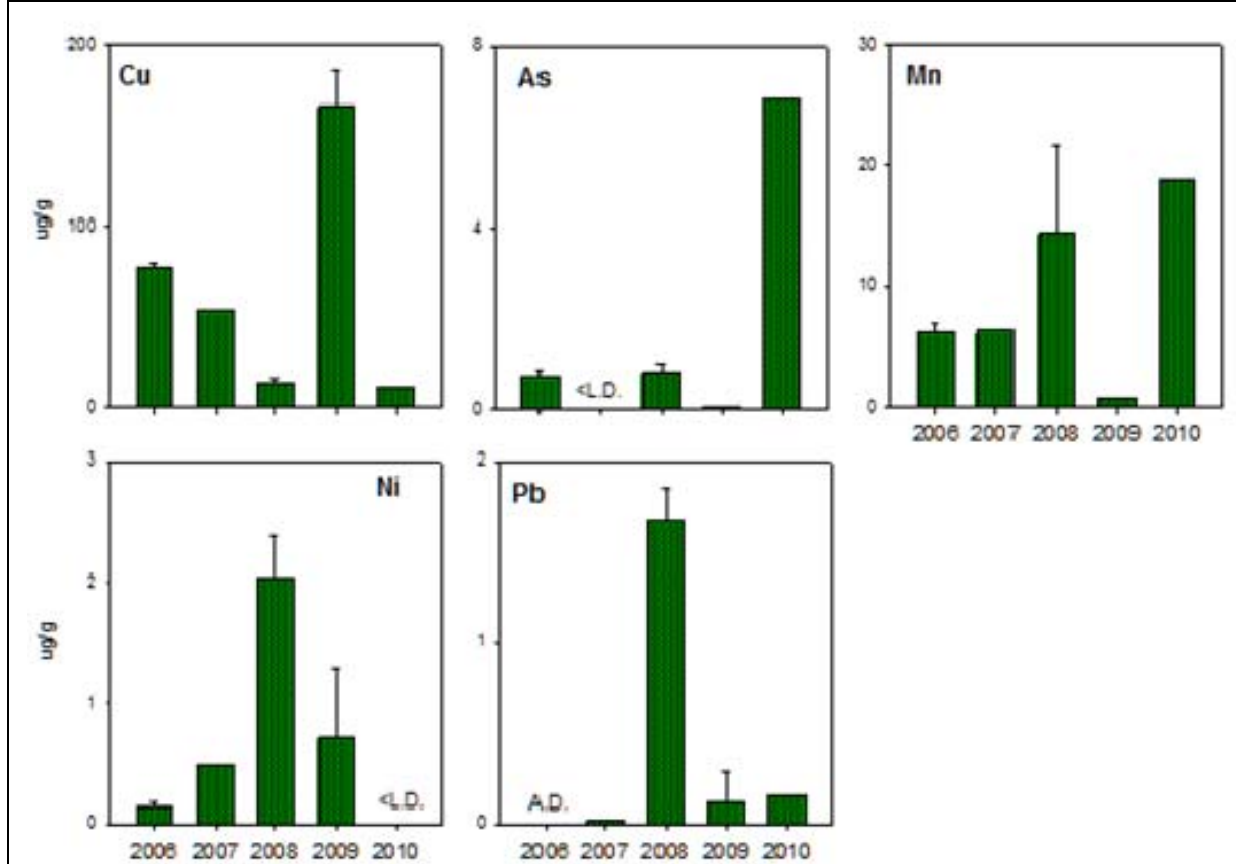

Figura 4 - Concentrações de metais ( $\mu \mathrm{g} / \mathrm{g}$ - peso seco) no camarão-rosa analisado por inteiro, entre os anos de 2006 e 2010. (A.D.: Ausência de dados, L.D.: Limite de detecção). 


\section{Aspecto de consumo humano}

Para comparação com as concentrações máximas de metais permitidas pela Legislação Brasileira, as concentrações de metais foram expressas em peso úmido, calculado com base em umidade média de $78,7 \%$ para o camarão inteiro e $79,7 \%$ para o camarão limpo.

Tanto para o camarão inteiro quanto para o limpo, foram encontradas concentrações de As acima do permitido pela legislação $\left(1 \mu \mathrm{g} \mathrm{g}^{-1}\right)$, no ano de 2010 (valor encontrado: $1,46 \mu \mathrm{g} \mathrm{g}^{-1}$, no camarão limpo e $1,55 \mu \mathrm{g} \mathrm{g}^{-1}$ no inteiro). O camarão inteiro também exibiu concentrações de $\mathrm{Cr}\left(0,14 \mu \mathrm{g} \mathrm{g}^{-1}\right)$ e de $\mathrm{Cu}\left(50,8 \mu \mathrm{g} \mathrm{g}^{-1}\right)$ acima dos limites estabelecidos pela legislação $\left(0,1 \mu \mathrm{g} \mathrm{g}^{-1}\right.$ para o $\mathrm{Cr}$, e $30 \mu \mathrm{g} \mathrm{g}^{-1}$ para o $\left.\mathrm{Cu}\right)$, nos anos de 2006 e em 2010, respectivamente.

\section{DISCUSSÃO}

\section{Metais no camarão-rosa}

As concentrações reduzidas no camarão limpo, quando comparado ao inteiro são resultados comumente encontrados na literatura entre espécies do gênero Penaeus (Pourang et al., 2005; Darmono \& Denton,1990; Paez-Osuna \& Tron-Mayen, 1996), e podem estar associadas ao fato da análise deste último incluir o hepatopâncreas (Yılmaz \& Yılmaz, 2007).

Nos crustáceos decápodes, o hepatopâncreas tem funções semelhantes ao fígado dos vertebrados, estas relacionadas ao metabolismo de nutrientes e elementos essenciais, e remoção de elementos não essenciais (Campbell, 2005). Com isso, assim como nos vertebrados, suas células podem concentrar quantidades de elementos bem mais altas em relação ao músculo (Chou et al., 2002; Çogun et al. 2005; Pourang et al., 2005 e Firat et al., 2008).

Adicionalmente, o exoesqueleto geralmente contém uma carga significativa de metais, dessa forma, elementos passivamente absorvidos neste tecido contribuirão para aumentar a concentração de metais no organismo (Rainbow, 2007).

\section{Comparações interanuais}

Alguns estudos mostram a influência de variações sazonais nas concentrações de metais nos tecidos de crustáceos, que são atribuídas aos ciclos biológicos internos do organismo. Como no presente estudo os camarões foram coletados no verão em todos os anos, foi assumido que a variabilidade encontrada foi resultante de variações no ambiente.

Fatores como mudanças na salinidade de áreas costeiras ao longo do ano dependendo da chuva ou do aporte de água doce dos rios contribuem para a variação na concentração dos metais. No caso de estuários, baixas salinidades são conhecidas por aumentar a biodisponibilidade de alguns metais (Nugegoda \& Rainbow, 1995).

As maiores concentrações de $\mathrm{Pb}$ nos organismos amostrados em 2008 corroboram o exposto acima, uma vez que o estuário encontrava-se com salinidade baixa, resultante do período chuvoso antecedente ao verão de 2008. Neste mesmo ano, as concentrações de $\mathrm{Pb}$ na água também apresentaram valores superiores aos demais anos (Tabela 1 ), indicando que as concentrações deste metal observadas no camarão-rosa também refletiram o maior aporte continental carreado pelos rios.

Já as maiores concentrações de As nos organismos coletados em 2010, apresentaram comportamento inverso às concentrações observadas na água estuarina, que exibiu os menores valores no ano de 2010.

O comportamento do As em águas estuarinas é complexo e ainda não foi completamente compreendido. Na fração particulada, o arsênio pode estar associado às partículas inorgânicas, principalmente minerais, como oxi-hidróxidos de ferro, que podem ser provenientes da ressuspensão do sedimento, erosão de solos, deposição (Anjos, 2006). Os maiores teores desse metal observados na água estuarina em 2008 podem 
estar associados à adsorção no material em suspensão, que apresentou valores elevados nesse ano. Como os teores determinados na água referem-se à concentração total de As, a sua fração assimilável pelos camarões pode ter sido pequena neste ano, uma vez que a biodisponibilidade deste metal depende da sua forma química.

Além de afetar a biodisponibilidade dos metais, flutuações na salinidade também podem ocasionar respostas fisiológicas nos organismos, alterando a absorção destes compostos (Wright, 1995). As altas concentrações de Mn observadas nos camarões inteiros amostrados nos verões de 2008 e em 2010, quando o estuário encontrava-se sob condições menos salinas, podem ser explicadas pela similaridade química deste elemento com o $\mathrm{Ca}$. O Mn compete com o Ca durante as carbonatações das carapaças (Phillips, 1977) e, uma vez exposto a um meio deficiente de $\mathrm{Ca}$, o organismo tende a substituí-lo por outros metais que apresentaram grande disponibilidade no meio, no caso, o Mn(Pourang et al., 2005; Rainbow, 1995; Hockett et al., 1997 ; Nam et al., 2005).

As maiores concentrações de $\mathrm{Cu}$ no camarão inteiro no verão de 2009 estão associadas à salinização do estuário. Contreira (2009) observou que o Cu lábil possui uma relação direta com a salinidade, e em situações de grande salinização, ocorre redução da concentração de ligantes, aumentando a disponibilidade desse elemento para a biota, corroborando os resultados encontrados neste estudo.

Já em condições de baixa salinidade, o Cu apresenta uma alta capacidade de complexação (Contreira, 2009). A alta concentração de matéria orgânica, como substâncias húmicas e fúlvicas, proveniente dos rios da bacia de drenagem da Lagoa dos Patos, fornece uma grande quantidade de sítios de ligação para o metal, diminuindo sua biodisponibilidade. Esta observação também ficou evidenciada neste estudo pelas menores concentrações de $\mathrm{Cu}$ encontradas nos organismos coletados nas safras de 2008 e 2010, quando o estuário encontrou-se em condições de baixa salinidade.

\section{Aspecto de consumo humano}

Os valores estabelecidos pela Legislação referem-se às concentrações totais dos elementos, no entanto, nem sempre refletem o risco potencial de contaminação. A toxicidade, biodisponibilidade e mobilidade de metais traço em águas naturais são mais afetadas pela forma como esses elementos ocorrem, ou seja, por sua especiação química, do que pela sua concentração total (Donat et al., 1994).

No caso do As, suas formas orgânicas são facilmente excretadas. Já as formas inorgânicas, em especial As (III) e As (V) são as espécies mais tóxicas, podendo causar doenças cardiovasculares, distúrbios no sistema nervoso central e vascular periférico e câncer de pele, entre outros efeitos adversos (Carvalho et al., 2004).

Já os compostos do $\mathrm{Cr}(\mathrm{VI})$ são extremamente tóxicos aos organismos, podendo causar, entre outros efeitos, lesões mutagênicas e carcinogênicas no fígado, pulmão e rins (Pechova \& Pavlata, 2007).

Em um estudo envolvendo a especiação química do $\mathrm{Cr}$ no estuário da Lagoa dos Patos, não foi encontrado $\mathrm{Cr}$ na sua forma lábil (Borges, 2009). Para o As, que também apresentou concentrações altas no camarão limpo, forma mais amplamente consumida pela população, ainda não há dados de especiação para o local de estudo, podendo portanto, apresentar algum risco aos consumidores, em função dos efeitos deletérios citados acima.

\section{Comparações com outros estudos}

Primeiramente, foi feita uma avaliação cronológica das concentrações de metais observadas no camarão-rosa, através da comparação dos resultados obtidos neste estudo, com os de Corradi (2002), que utilizou a mesma espécie, coletada também no estuário da Lagoa dos Patos. 
GARCIA, J. G.; NIENCHESKI, L. F. H. Avaliação temporal da acumulação de elementos traço no camarão-rosa Farfantepenaeus paulensis no estuário da Lagoa dos Patos, RS, Brasil.

Para comparação de ambos os trabalhos, foram utilizados somente os dados do verão de 2009, uma vez que foi o ano com as condições hidrológicas mais próximas às de Corradi (2002).

Com exceção do $\mathrm{Fe}$, que se apresentou significativamente maior no presente estudo, os demais metais exibiram concentrações significativamente menores que os valores encontrados em Corradi (2002) (Tabela 4).

Tabela 4 - Médias ( $\mu \mathrm{g} \cdot \mathrm{g}^{-1}$ - peso seco) de metais observados no camarãorosa em dois estudos cronologicamente distintos, e resultados da aplicação do teste $t$ de Student.

\begin{tabular}{c|cccc}
\hline Metais & Corradi (2002) & Presente estudo & $\mathrm{t}$-value & $\mathrm{p}$ \\
\hline $\mathrm{Cd}$ & 0,273 & 0,045 & 1,571 & 0,0002 \\
$\mathrm{Cr}$ & 0,447 & 0,047 & 2,015 & 0,0201 \\
$\mathrm{Cu}$ & 47,212 & 24,881 & 1,604 & 0,0037 \\
$\mathrm{Fe}$ & 9,109 & 72,663 & $-4,436$ & 0,0013 \\
$\mathrm{Mn}$ & 2,729 & 0,454 & 1,967 & 0,0006 \\
$\mathrm{~Pb}$ & 0,364 & 0,175 & 3,079 & 0,0117 \\
$\mathrm{Zn}$ & 70,480 & 35,030 & 3,196 & 0,0096 \\
\hline
\end{tabular}

As menores concentrações observadas nos organismos deste estudo, quando comparadas a Corradi (2002) poderiam levar a inferência de que estuário sofreu uma redução no lançamento de contaminantes ao longo desses 10 anos. No entanto, sabe-se que nesse intervalo de tempo, houve um grande acréscimo de atividades potencialmente poluidoras no entorno da Lagoa dos Patos, conforme citado na introdução.

Dessa forma, as diferenças encontradas podem estar relacionadas aos diferentes locais de coleta de ambos os estudos, dentro do estuário. Tendo em vista que os organismos foram coletados no Saco da Mangueira no ano de 2000, região do estuário de baixa hidrodinâmica comparada ao local de coleta do presente estudo, os contaminantes possuem um maior tempo de residência e podem ter sido removidos para o sedimento de fundo, representando uma fonte de contaminação maior que no local onde a hidrodinâmica é alta, onde pode ocorrer fenômeno inverso, e os contaminantes serem liberados a partir do sedimento para a coluna d'água.

Adicionalmente, no local de coleta do presente estudo ocorre "circulação" de organismos, o que pode ter feito com que o tempo de permanência dos camarões dentro do estuário no ano de 2009, tenha sido bem menor com relação aos organismos amostrados por Corradi (2002).

A Tabela 5 apresenta concentrações de metais encontradas em camarões pertencentes ao gênero Penaeus em outros locais. Os valores apresentados na Tabela 4 para este estudo são valores médios de todos os anos amostrados.

Tabela 5 - Concentração de elementos-traço ( $\mu \mathrm{g} \mathrm{g}^{-1}$ - peso seco) em espécies do gênero Penaeus.

\begin{tabular}{|c|c|c|c|c|c|c|c|c|c|c|c|}
\hline Espécie & Local & L (cm) & Cd & $\mathrm{Cr}$ & $\mathbf{C u}$ & $\mathbf{F e}$ & Mn & $\mathbf{N i}$ & $\mathbf{P b}$ & $\mathbf{Z n}$ & Referência \\
\hline $\begin{array}{c}\text { Farfantepenaeus } \\
\text { paulensis }\end{array}$ & $\begin{array}{l}\text { Estuário da Lagoa } \\
\text { dos Patos (Brasil) }\end{array}$ & 3,8 & 0,07 & 0,12 & 19,99 & 47,55 & 2,63 & 0,19 & 0,34 & 33,99 & Presente estudo \\
\hline $\begin{array}{c}\text { Farfantepenaeus } \\
\text { paulensis* }\end{array}$ & $\begin{array}{r}\text { Estuário da Lagoa } \\
\text { dos Patos (Brasil) }\end{array}$ & 3,8 & 0,22 & 0,21 & 64,42 & 136,43 & 9,3 & 0,68 & 0,49 & 46,36 & Presente estudo \\
\hline Penaeus vannamei & Costa mexicana do Pacífico & 8,5 & 0,29 & 1,06 & 18,3 & 224,1 & 4,77 & 1,28 & --- & 62,5 & Paez-Osuna \& Ruiz Fernandes, 1995 \\
\hline Penaeus semisulcatus & $\begin{array}{l}\text { Iskenderun Bay } \\
\text { (Turquia) }\end{array}$ & 11,84 & 16,72 & 60,38 & 34,24 & 18,69 & --- & --- & --- & 27,75 & Firat et al, 2008 \\
\hline Parapenaeus longirostris & $\begin{array}{l}\text { Mar de Marmara } \\
\text { (Turquia) }\end{array}$ & 9,64 & 0,77 & --- & 24,5 & 56,2 & 2,9 & 4,27 & 4,82 & 40,42 & Kurun et al., 2007 \\
\hline Litopenaeus stylirostris & Golfo da California & 17,6 & 0,66 & --- & 25,4 & ---- & --- & --- & 5,3 & 57,8 & Frías-Espiricueta et al., 2007 \\
\hline Xiphopenaeus Kroyeri* & Baía de Ubatuba & 16,3 & 4,14 & 3,86 & 72,34 & --- & -- & --- & 68,3 & 108,1 & Mantelatto et al., 1999 \\
\hline
\end{tabular}

$*(*)$ : camarão inteiro; L: comprimento total 
Com relação à fração abdominal, os valores de $\mathrm{Cu}, \mathrm{Zn}$ e $\mathrm{Mn}$ apresentaram as menores flutuações entre os estudos, o que se justifica pelo fato de serem metais essenciais, portanto pouca variabilidade interespecífica é esperada (Savinov et al., 2003). Já os metais $\mathrm{Cd}, \mathrm{Cr}, \mathrm{Ni}$ e $\mathrm{Pb}$ apresentaram concentrações bem menores neste estudo, comparado aos demais.

Comparando-se os valores encontrados para o camarão inteiro no estuário da Lagoa dos Patos com os valores encontrados por Mantellato et al.(1999) Xiphopenaeus kroyeri também analisado por inteiro, observa-se que este último apresentou concentrações superiores para todos os metais, especialmente para o $\mathrm{Pb}$.

Observou-se que os organismos analisados no presente estudo apresentaram tamanhos bem inferiores aos demais a que estão sendo comparados, e dessa forma, esta variável poderia estar influenciando nos menores teores de metais nos organismos deste estudo. No entanto, há pouca informação sobre a relação entre esta variável e níveis de metais em crustáceos e, dentre os estudos realizados, observa-se uma relação inversa entre tamanho e concentração de metais, devido ao fato de que animais jovens e menores tendem a ingerir uma quantidade maior de alimentos, em função da elevada atividade metabólica e, conseqüentemente, mais contaminantes, caso estes estejam presentes (Mantelatto, 1999). A comparação deste estudo com os demais mostrou resultados contrários aos encontrados na literatura, uma vez que o presente estudo apresentou teores de metais nos organismos inferiores aos demais estudos, onde os organismos analisados apresentaram tamanhos maiores.

Dessa forma, as diferenças encontradas podem então, estar relacionadas a distintos hábitos alimentares, uma vez que a alimentação representa uma parte essencial na absorção de metais (Coetzee et al., 2002).

Adicionalmente, distintas fontes poluidoras, bem como diferenças nas condições ambientais incluindo parâmetros físico-químicos entre os locais também podem ter contribuído para as diferenças encontradas.

\section{CONCLUSÕES}

1. O camarão analisado por inteiro exibiu, em geral, maiores teores de metais que a fração abdominal, e apresentou concentrações de $\mathrm{Cr}$, $\mathrm{Cu}$ e As acima do limite estabelecido pela Legislação Brasileira, sendo que o As ultrapassou o limite, também, na fração abdominal. Estes valores estabelecidos pela legislação são um indicativo do estado de contaminação do organismo, e adicionalmente, estudos de especiação representam uma ferramenta completa para avaliações ecotoxicológicas.

2. Os camarões limpos apresentaram maiores teores de As e de $\mathrm{Cr}$, em anos de maior ocorrência de pluviosidade, associada ao fenômeno El Niño. Já os camarões inteiros apresentaram maior sensibilidade às variações ambientais anuais ocorridas no estuário, onde, além das variações nas concentrações de $\mathrm{As}$ e $\mathrm{Pb}$, também apresentaram concentrações de $\mathrm{Mn}$ relacionadas inversamente à salinidade. Contrariamente, as maiores concentrações de $\mathrm{Cu}$ no camarão inteiro foram observadas quando o estuário apresentou-se dominado por água marinha, característico de períodos de La Niña.

3. Os demais metais analisados nos organismos não estiveram relacionados com os parâmetros ambientais contemplados neste estudo.

\section{REFERÊNCIAS}

ALTMAYER, F. Pescadores artesanais do estuário da Lagoa dos Patos - RS: Uma análise de sua percepção do meio natural como subsídio para um projeto de educação ambiental. Rio Grande, 1999. 159f. Dissertação de Mestrado. Universidade Federal do Rio Grande.

ANJOS, V. E. Especiação de cobre e arsênio no complexo Estuarino da baía de Paranaguá. Curitiba, 2006. 175f. Dissertação de mestrado, Universidade Federal do Paraná. 
ANVISA. Agência Nacional de Vigilância Sanitária.1998. Portaria nº 685, de 27 de agosto de 1998.

BARBIERI, E.; PASSOS, E. A.; GARCIA, C. A. Use of metabolism to evaluate the sublethal toxicity of mercury on Farfantepaneus brasiliensis larvae (Latreille 1817, Crustacean). Journal Shellfish Research, v. 24, n. 4, p. 1229-1234, 2005.

BAUMGARTEN, M. G. Z.; NIENCHESKI, L. F. O estuário da Lagoa dos Patos: Variações de alguns parâmetros físico-químicos da água e metais associados ao material em suspensão. Ciência e Cultura, v. 42, p. 390-396, 1990.

BERLATO, M. A.; FONTANA, D.C. El Niño e La Niña: impactos no clima, na vegetação e na agricultura do Rio Grande do Sul; aplicações de previsões climáticas na agricultura. Porto Alegre: UFRGS, 2003.

CARVALHO, C. E. V.; CAVALCANTE, M .P. O. ; GOMES, M. P. ; FARIA, V. V.; REZENDE, C. E. Heavy Metal distribution in Mussel (Perna perna, L.) from Santana Island, Macaé, SE, Brazil. Ecotoxicology and Environmental Restoration, v. 4, n. 10, p. 1-5, 2001.

CHOU, C. L.; PAON, L. A.; MOFFATT, J. D. Cadmium, copper, manganese, silver, and zinc in rock crab (Cancer irroratus) from highly copper contaminated sites in the Inner Bay of Fundy, Atlantic Canada. Bulletin of Environmental Contamination and Toxicology, v. 68 , p. $885-892,2002$.

COETZEE, L.; DU PREEZ, H. H.; VAN VUREN, J. H. J. Metal Concentration in Clarias gariepinus and Labeo umbratus from the Olifants and Klein Olifant River, Mpumalanga, South Africa: Zinc, copper manganese, lead, chromium, nickel, aluminium and iron. South Africa, 2002. Rand Afrikaans University.

CONTREIRA, L. P. Especiação química de cobre em ambientes aquáticos próximos ao município de Rio Grande, RS. Rio Grande, 2009. 68f. Dissertação de mestrado, Universidade Federal do Rio Grande.

CORRADI, C. Avaliação das condições do litoral do Rio Grande do Sul através da bioacumulação de metais pesados por mexilhões, por cracas e por camarões no estuário da Lagoa dos Patos. Rio Grande, 2002. 82f. Dissertação de mestrado. Universidade Federal de Rio Grande.

ÇOGUN, H. Y.; YUZEREROGLU, T. A.; KARGIN, F.; FIRAT, Ö. Seasonal variations and tissue distribution of heavy metals in shrimp and fish species from the Yumurtalik Coast of Iskenderun Gulf, Mediterranean. Bulletin of Environmental Contamination and Toxicology, v.75, p. 707-715, 2005.

DARMONO, D.; DENTON, G. R. W. Heavy metal concentrations in the banana prawn, Penaeus merguiensis, and leader prawn, Penaeus monodon, in the Townsville region of Australia. Bulletin of Environment Contamination and Toxicology, v.44, p.479-486, 1990.

D'INCAO, F. Taxonomia, padrões distribucionais e ecológicos dos Dendobranchiata (Crustacea: Decapoda) do Brasil e Atlântico Ocidental. Rio grande, 1995. 364f. Tese de Doutorado. Universidade Federal do Paraná.

D'INCAO, F. Pesca e biologia de Penaeus paulensis na Lagoa dos Patos, RS. Atlântica, Rio Grande, v. 13, n. 1, p. 159-169, 1991.

D'INCAO, F.; VALENTINI, H.; RODRIGUES, L. F. Avaliação da pesca de camarões nas regiões Sudeste e Sul do Brasil. 1965-1999. Atlântica, v. 24, n. 2, p. 103-116, 2002.

DONAT, J. R.; LAO, K. A.; BRULAND, K. W. Speciation of dissolved copper and nickel in south San Francisco Bay: a multi-method approach. Analytica Chimica Acta, v. 284, p. 547-571, 1994.

FERNANDES, E. H. L., DYER, K. R.; MÖLLER, O.; NIENCHESKI, L. F. H. The Patos Lagoon Hydrodynamics during an El Niño event. Continental Shelf Research, v. 22, p. 16991713, 2002. 
FERNANDES, E. H. L. Modeling of the hydrodynamics of the Patos Lagoon, Brazil. Plymouth, 2001. 198f. Tese de Doutorado, Universidade de Plymouth, UK.FIRAT, Ö.; GÖK, G.; ÇOĞUN, H. Y.; YÜZEREROĞLU, T. A.; KARGIN, F. Concentrations of Cr, Cd, Cu, $\mathrm{Zn}$ and $\mathrm{Fe}$ in crab Charybdis longicollis and shrimp Penaeus semisulcatus from the Iskenderun Bay, Turkey. Environmental Monitoring and Assessment, v. 147, p. 117-123, 2008.

FRÍAS-ESPERICUETA, M. G.; IZAGUIRRE-FIERRO, G.; VALENZUELA-QUIÑONEZ, F.; OSUNA-LÓPEZ, J. I.; VOLTOLINA, D.; LÓPEZ-LÓPEZ, G.; MUY-RANGEL, M. D.; RUBIOCASTRO, W. 2007. Metal Content of the Gulf of California Blue Shrimp Litopenaeus stylirostris (Stimpson). Bulletin of Environmental Contamination Toxicology, v. 79, p. 214-217.

GARCIA, A. M.; VIEIRA, J. P. O aumento da diversidade de peixes no estuário da lagoa dos Patos durante o episódio El Niño 1997-1998. Atlântica, v. 23, p. 133-152, 2001.

HOCKETT, D, INGRAM P, LEFURGEY A. Strontium and manganese uptake in the barnacle shell: electron probe microanalysis imaging to attain fine temporal resolution of biomineralization activity. Marine Environment Research, v.43, p.131-143, 1997.

IWAI, M. Desenvolvimento larval e pós-larval de Penaeus (Melicertus) paulensis Pérez Farfante, 1967 (Crustacea, Decapoda) e o ciclo de vida dos camarões do gênero Penaeus da região centro-sul do Brasil. São Paulo, 1978. 137f. Tese de doutorado, Universidade de São Paulo.

KURUN, A.; BALKIS, H.; BALKIS, N. Accumulations of total metal in dominant shrimp species (Palaemon adspersus, Palaemon serratus, Parapenaeus longirostris) and bottom surface sediments obtained from the Northern Inner Shelf of the Sea of Marmara Environmental Monitoring and Assessment, v. 135, p. 353-367, 2007.

MANTELATTO, F. L. M.; AVELAR, W. E. P; TOMAZELLI, A. V.; SILVA, D. M. L; LOPEZ, J. L. C; SHUHAMA, T. Heavy metals in the shrimp Xiphopenaeus kroyeri (Heller, 1862). (Crustacea, Penaeidae) from Ubatuba Bay, São Paulo, Brazil. Bulletin of Environmental and Toxicology, v.62, n.2, p.152-159, 1999.

MÖLLER, O.; FERNANDES, E. Hidrologia e Hidrodinâmica. In: O Estuário da Lagoa dos Patos: Um século de transformações. Seeliger, U.; Odebrecht, C. (Eds.). $1^{\text {a }}$ ed. Rio Grande : FURG. 2010. 180 p.

MÖLLER . O.; PAIM, P. S. G.; SOARES, I. D. Facteurs et mechanismes de la circulation des eaux dans l'estuarie de la Lagune dos Patos (RS, Bresil). Bull. Inst. Geol. Basin Aquitaine (Bordeaux). v. 49, p. 15-21, 1991.

NAM, D. H.; ANAN, Y.; IKEMOTO, T.; OKABE Y, KIM EY, SUBRAMANIAN A, SAEKI K, TANABE S. Specific accumulation of 20 trace elements in great cormorants (Phalacrocorax carbo) from Japan. Environmental Pollution, v. 134, p. 503-514, 2005.

NIENCHESKI, L. F.; BAUMGARTEN, M. G. Z. Crustacea, Cirripedia (Cracas). In: Avaliação Ambiental de Estuários Brasileiros: Diretrizes Metodológicas. Lana, P.C.; Bianchini, A.; Ribeiro, C.A.O.; Niencheski, L.F.; Fillmann, G. \& Santos, C.S.G. (Organizadores). Projeto RECOS: Uso e apropriação de Recursos Costeiros. Grupo Temático: Biodiversidade e Qualidade Ambiental. Rio de Janeiro, Museu Nacional, 2006.

NUGEGODA, D.; RAINBOW, P. S. The uptake of dissolved zinc and cadmium by the decapod crustacean Palaemon elegans. Marine Pollution Bulletin, v. 31, p. 460-463, 1995.

PAEZ-OSUNA, F.; RUIZ-FERNANDES, C. Trace metals in the mexican shrimp Penaeus vannamei from estuarine and marine environments. Environmental Pollution, v. 87, p. 243-247, 1995.

PAEZ-OSUNA, F.; TRON-MAYEN, L. 1996. Concentration and distribution of heavy metals in tissues of wild and farmed shrimps Penaeus vannamei from the northwest coast Mexico. Environmental International, v. 22, p. 209-215, 1996. 
PECHOVA. A.; PAVLATA, L. Chromium as an essential nutrient: a review. Veterinary Medicine, v. 52, n. 1, p. 1-18, 2007.

PEREIRA, N. Influência dos fatores meteorológicos na salinidade e na abundância da safra de camarão-rosa, Farfantepenaeus paulensis pérez farfante, 1967, no estuário da Lagoa dos Patos, Sul do Brasil. Rio Grande, 2010. 118f. Dissertação de mestrado, Universidade Federal do Rio Grande.

PHILLIPS, D. J. H. The use of biological indicator organisms to monitor trace metal pollution in marine and estuarine environments: a review. Environment Pollutions, v. 13, p. 281-317, 1977.

POURANG, N.; DENNIS, J. H.; GHOURCHIAN, H. Distribution of heavy metals in Penaeus semisulcatus from Persian Gulf and possible role of metallothionein in their redistribution during storage. Environmental Monitoring and Assessment, v. 100, p. 71-88, 2005.

RAINBOW, P. S. Trace metal bioaccumulation: models, metabolic availability and toxicity. Environment International, v. 33, p. 576-582, 2007.

SAVINOV, V. M.; GABRIELSEN, G. W.; SAVINOVA, T. N. Cadmium, zinc, copper, arsenic, selenium and mercury in seabirds from the Barents Sea: levels, inter-specific and geographical differences. The Science of the Total Environment, v. 306, p. 133-158, 2003.

SUPERINTENDÊNCIA DO PORTO DE RIO GRANDE (SUPRG). Relatórios Técnicoscientíficos. Acessado em 2010. Disponível em: http://www.portoriogrande.com.br

USTRA, J. R. Concentrações de mercúrio na biota e sedimentos no sul do estuário da Lagoa dos Patos. Rio Grande, 2001. Dissertação de mestrado, Universidade Federal do Rio Grande.

VILAS BOAS, D. Distribuição dos sais, nutrientes, elementos maiores e metais pesados na Lagoa dos Patos, RS. Rio Grande, 1990. Dissertação de mestrado, Universidade Federal do Rio Grande.

YILMAZ, A. B.; YILMAZ, L. Influences of sex and seasons on levels of heavy metals in tissues of green tiger shrimp (Penaeus semisulcatus de Hann, 1844). Food Chemistry, v. 101, p.1664-1669, 2007.

WRIGHT, D.A. Trace metal and major ion interactions in aquatic animals. Marine Pollution Bulletin, v. 31, p. 8-18, 1995.

ZAR, J. H. Biostatistical Analysis. Prentice-Hall, Inc. New Jersey. USA. 1984, 718p. 